\title{
Analyse des pertes de puissance dans les transmissions par engrenages
}

\author{
Yasser Diab, Fabrice Ville ${ }^{a}$, Christophe Changenet et Philippe Velex \\ LaMCoS, INSA-Lyon, CNRS UMR 5259, 69621 Villeurbanne Cedex, France
}

Reçu le 7 mars 2006, accepté le 18 mai 2007

\begin{abstract}
Résumé - Les pertes de puissance dans les transmissions par engrenages grandes vitesses sont dues au frottement entre les dents, au procédé de lubrification (barbotage ou jet d'huile), au pompage d'un mélange air-huile durant l'engrènement et aux pertes par ventilation de la denture. Des approches complémentaires ont été développées afin de mieux évaluer ces pertes. Cette présentation les résume et propose une analyse des pertes pour deux exemples caractéristiques de transmissions par engrenage.
\end{abstract}

Mots clés : Transmission / engrenages / pertes de puissance / frottement / ventilation / piégeage

\begin{abstract}
Investigations on the power losses in gear transmissions. Power losses in high-speed gears come from the friction between the teeth, the lubrication process (dip or jet lubrication), the pumping of a gas-lubricant mixture during the meshing and the losses associated with windage effects. Different approaches have been developed in order to analyse the contribution of each power loss source. This presentation aims to describe these different approaches and to illustrate their potential with reference to two given geared transmission examples.
\end{abstract}

Key words: Transmissions / gears / power losses / friction / windage / pumping

\section{Introduction}

D’une manière générale, les pertes de puissance dans les transmissions par engrenages au niveau des engrènements peuvent être subdivisées en trois contributions : a) celle liée au frottement à la denture, b) celle due au phénomène de piégeage du mélange air-lubrifiant dans l'espace inter-dents et enfin c) celle induite par la ventilation de la denture.

Il existe peu de modèles dans la littérature permettant d'identifier ces différentes pertes de façon relativement précise. Les auteurs ont donc développé des modèles spécifiques pour évaluer celles-ci. Pour chacun de ces modèles, une étude expérimentale associée a permis d'en faire une première validation.

Cet article résume les trois modèles développés pour les trois sources de pertes ainsi que leur validation expérimentale. Pour plus de détails concernant les développements théoriques, le lecteur pourra se référer aux travaux cités en références. Ces modèles sont ensuite

\footnotetext{
${ }^{a}$ Auteur pour correspondance :

fabrice.ville@insa-lyon.fr
}

appliqués à deux réducteurs afin d'évaluer la contribution de chacune des pertes à la perte de puissance totale.

\section{Pertes par ventilation de la denture [1]}

Ces pertes qui correspondent à la puissance perdue du fait de la traînée aérodynamique de la denture dans le mélange air-huile d'un carter de réducteur, sont prépondérantes pour les transmissions par engrenages dits à grandes vitesses (vitesses périphériques supérieures à 90-120 m.s $\left.{ }^{-1}\right)$.

\subsection{Développements théoriques}

Deux approches théoriques ont été développées [1] :

a) l'une est basée sur une analyse dimensionnelle des différents facteurs significatifs permettant d'exprimer le couple de traînée total comme le produit de nombres adimensionnels, tels que le nombre de Reynolds, établis à partir de l'analyse des paramètres considérés comme les plus influents :

1/ paramètres géométriques associés au mécanisme (largeur de denture, rayon primitif, nombre de dents), 


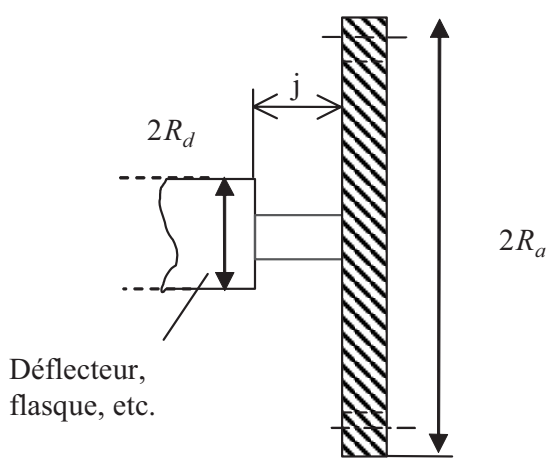

Roue dentée

Fig. 1. Paramètres géométriques pour l'influence de déflecteurs, flasques et/ou obstacles.

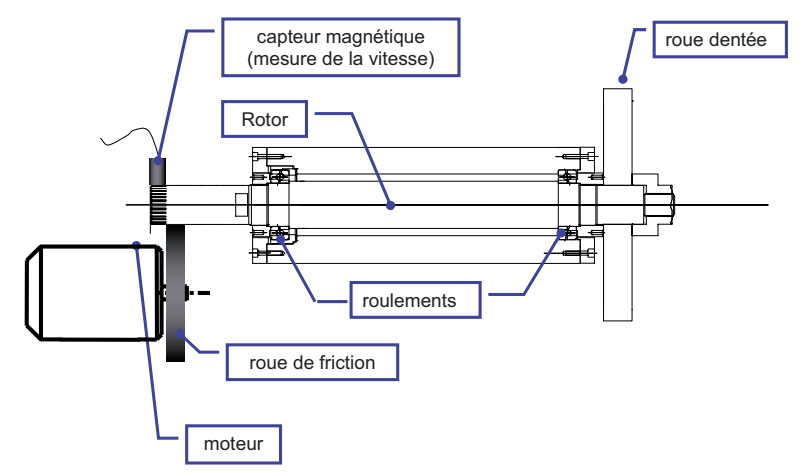

Fig. 2. Dispositif expérimental.

2 / paramètres liés au fluide (viscosité et masse volumique),

$3 /$ paramètres prenant en compte la présence de flasques, figure 1 (rayon du déflecteur et distance entre le déflecteur et la roue dentée),

4/ paramètre cinématique (vitesse de rotation).

Les coefficients liés aux différents groupes adimensionnés ont été obtenus à partir de nombreux essais menés sur le dispositif expérimental développé pour cette étude.

b) la seconde approche est basée sur une analyse simplifiée des débits autour des roues dentées en rotation. En s'inspirant des études de Dawson [1], il apparaît que la perte de puissance est due à la perte par ventilation sur les côtés de la roue dentée et à la perte par ventilation de la denture. À partir de cette constatation, une formule donnant le couple de traînée total a été développée à partir des équations de la mécanique des fluides [2].

\subsection{Validation des modèles}

Les deux approches ont été comparées avec les résultats expérimentaux issus d'un banc développé spécifiquement pour cette étude.

Ce dispositif expérimental (Fig. 2) est constitué d'un moteur électrique de $3 \mathrm{~kW}$ qui entraîne en rotation, par l'intermédiaire d'une roue de friction, un arbre supporté par deux roulements de haute précision pré-chargés. La
Tableau 1. Caractéristiques des roues à dentures droites testées.

\begin{tabular}{|c|c|c|c|}
\hline $\mathrm{N}^{\circ}$ de roue dentée & $\begin{array}{c}\text { diamètre primitif } \\
(\mathrm{mm})\end{array}$ & $\begin{array}{c}\text { largeur } \\
(\mathrm{mm})\end{array}$ & $\begin{array}{c}\text { module } \\
(\mathrm{mm})\end{array}$ \\
\hline 1 & 288 & 30 & 4 \\
\hline 2 & 144 & 30 & 4 \\
\hline 3 & 144 & 60 & 4 \\
\hline 4 & 144 & 60 & 6 \\
\hline
\end{tabular}

roue dentée est montée à l'une des extrémités du rotor, l'autre extrémité est rainurée afin de pouvoir mesurer la vitesse de rotation de l'arbre grâce à un capteur magnétique. Une fois que la vitesse maximale est atteinte, l'ensemble arbre-roue dentée est désolidarisé du système d'entraînement au moyen d'un vérin pneumatique. Connaissant l'inertie de l'ensemble, la loi de décroissance en vitesse fournit la puissance dissipée au cours du temps. Des études expérimentales ont été menées pour 4 roues dentées de caractéristiques différentes (Tab. 1). L'ensemble des résultats expérimentaux comparés aux résultats fournis par les différents modèles est présenté à la figure 3 .

L'accord entre les résultats expérimentaux et les formules issues des analyses dimensionnelles et des débits est très bon (l'écart relatif n'excède pas 14 \%).

\section{Pertes par piégeage [3]}

Dans les transmissions par engrenages, la lubrification de la denture est nécessaire pour refroidir les surfaces et réduire le frottement. Cependant, pour des engrenages droits ou hélicoïdaux à grandes vitesses, il semble que le mélange air-lubrifiant présent dans l'espace interdents serait successivement compressé et détendu provoquant des échauffements significatifs ainsi que des pertes de puissance [3-5]. Durant ces phases de compressionextension, une fraction du mélange air-lubrifiant est expulsée de l'espace inter-dents. La durée d'une période d'engrènement étant extrêmement courte dans les turboréducteurs, le mélange air-lubrifiant peut être compressé et chauffé de façon significative. Cette phase de compression est ensuite suivie d'une phase d'aspiration lorsque le volume inter-dents croît de nouveau lors du dégrènement. La perte de puissance totale correspond à l'énergie échangée avec le milieu ambiant lors des phases de compression et d'extension.

\subsection{Développements théoriques}

Un modèle hydrodynamique approché du piégeage du mélange air-lubrifiant dans l'espace inter-dents d'engrenages à dentures droites ou hélicoïdales a été développé [3]. Il repose sur i) la résolution de l'équation de continuité des débits massiques combinée au premier principe de la thermodynamique appliqué à un système ouvert, ii) la définition en fonction du temps de la variation du volume 


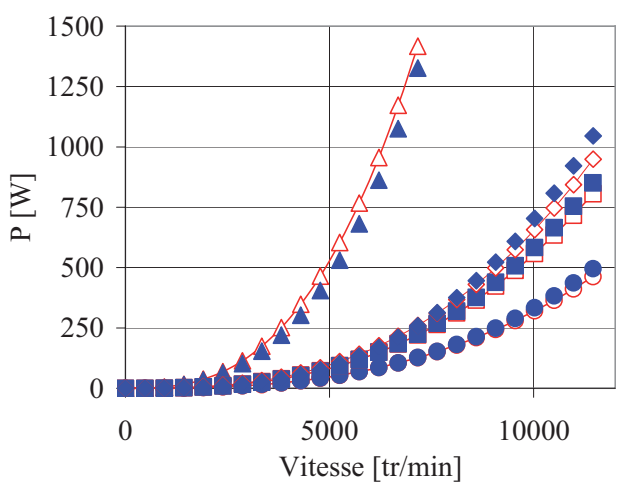

formule issue de l'analyse dimensionnelle [1]

$$
\begin{aligned}
& -\checkmark \text { roue dentée } n^{\circ} 1 \text { (théorie) } \\
& -\circ \text { roue dentée } n^{\circ} 2 \text { (théorie) } \\
& -\square-\text { roue dentée } n^{\circ} 3 \text { (théorie) } \\
& -\checkmark \text { roue dentée } n^{\circ} 4 \text { (théorie) }
\end{aligned}
$$

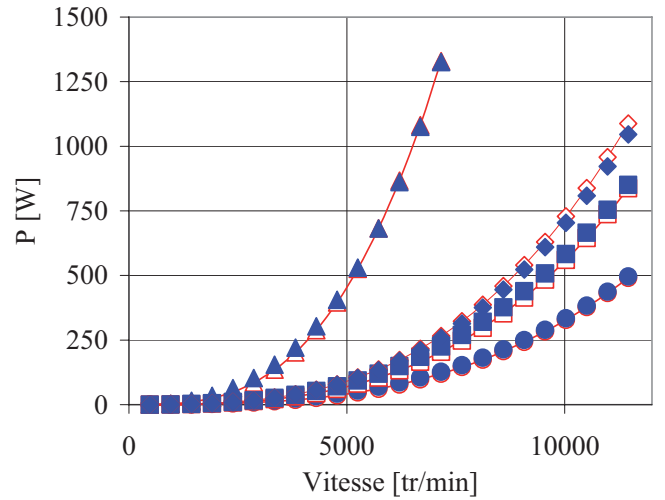

formule issue de l'analyse des débits [1]

$\Delta$ roue dentée $\mathrm{n}^{\circ} 1$ (expériences) roue dentée $n^{\circ} 2$ (expériences)

- roue dentée $\mathrm{n}^{\circ} 3$ (expériences)

- roue dentée $n^{\circ} 4$ (expériences)

Fig. 3. Comparaisons entre les résultats expérimentaux et les différents modèles.

inter-dents, iii) la description géométrique des surfaces de fuites axiales et radiales.

Un système ouvert (volumes discrets des espaces interdents découpés en tranches) est délimité par une enveloppe, éventuellement déformable, possédant au moins une ouverture qui autorise un transfert de matière (débit massique). En supposant que le frottement entre le fluide et les parois peut être négligé et que les vitesses du fluide dans les orifices sont suffisamment élevées, l'opération de compression-expansion entre les dentures peut être assimilée à un processus isentropique (réversible et adiabatique).

Il est à noter que, dans les turboréducteurs, les vitesses d'expulsion du mélange air-lubrifiant peuvent atteindre la vitesse du son et qu'il a donc fallu prendre en compte ce phénomène qui a pour effet de limiter les débits massiques.

Un schéma numérique a été mis en place en discrétisant les équations d'état par la méthode des différences finies et en les résolvant selon un schéma de prédiction/correction. Il est alors possible de calculer les niveaux de pression dans les volumes inter-dents et par conséquent les débits massiques et donc les pertes.

\subsection{Validation expérimentale}

Les résultats numériques ont pu être comparés à des valeurs expérimentales issues du banc d'essais développé par Houjoh [3]. Ce dispositif permet de mesurer, au cours de l'engrènement, la pression dans un volume inter-dent. Des mesures ont été effectuées pour différentes vitesses et différents angles d'hélice selon la largeur de la denture. Seules les comparaisons effectuées pour les engrenages à dentures droites pour des mesures de pression effectuées au milieu de la largeur de denture et pour une vitesse sont présentées à la figure 4. Les autres comparaisons sont

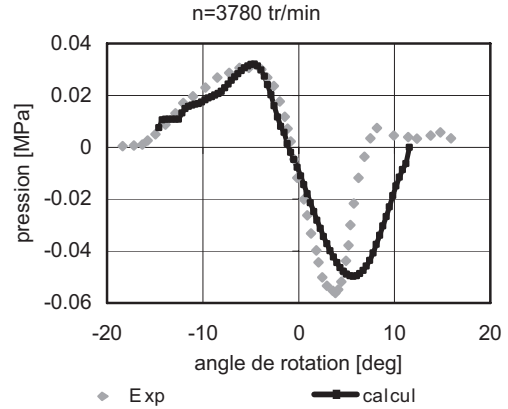

Fig. 4. Pressions mesurées et calculées au milieu de la largeur de denture (denture droite).

fournies dans [3]. L'ensemble des résultats montre un bon accord entre les pressions calculées et celles mesurées.

\subsection{Calcul des pertes}

La perte de puissance totale du système est égale au transfert de chaleur entre le système et son environnement. Ainsi, une fois que les pressions dans les volumes inter-dents sont bien évaluées, il est possible de calculer la perte de puissance moyenne sur une période d'engrènement (Fig. 5). Il apparaît qu'il y a une dépendance forte avec la vitesse de rotation puisque la perte de puissance est approximativement proportionnelle à la vitesse à la puissance 2,8 . L'angle d'hélice est également un paramètre de premier ordre.

\section{Pertes par frottement [6]}

Le frottement entre les dents d'engrenages est reconnu comme une des sources principales de perte de puissance dans les transmissions par engrenages ainsi 


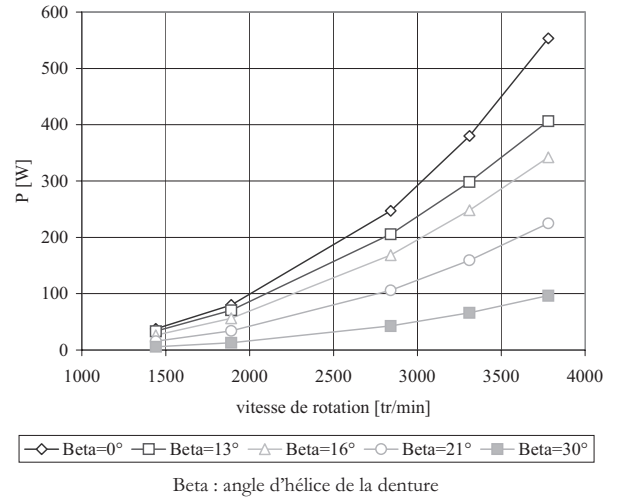

Fig. 5. Pertes de puissance dues au piégeage (dentures droites et hélicoïdales).

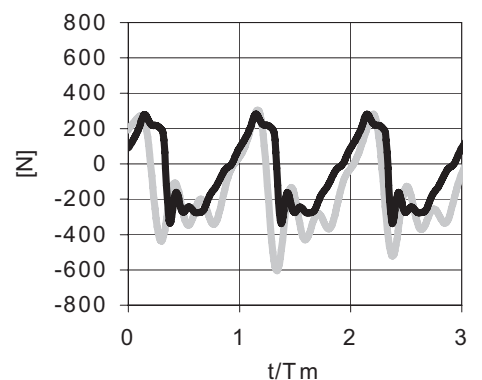

expériences $\quad$ modèle

Fig. 6. Force de frottement totale à l'engrènement calculée et

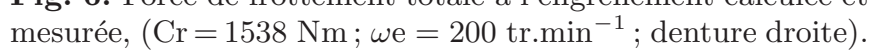

qu'une source potentielle de vibration et de bruit $[7,8]$. Pour des contacts lubrifiés, diverses lois de frottement empiriques basées sur des résultats de simulateurs peuvent être trouvées dans la littérature. Une de leurs limitations vient de la rugosité de surface des éprouvettes qui est souvent bien différente de celle d'engrenages réels. Ainsi, les auteurs ont dans un premier temps réalisé des mesures de frottement à l'aide d'un simulateur [6] avec des conditions de contact et une finition des éprouvettes proches de celles d'engrenages réels. Une nouvelle loi de frottement a été ensuite établie et intégrée dans un modèle du comportement dynamique de transmission par engrenages [9].

\subsection{Validation expérimentale}

Afin de valider le modèle du comportement dynamique des engrenages et la nouvelle loi établie, des mesures ont été réalisées sur un banc d'essai fortement instrumenté [10]. Ainsi, la force de frottement globale instantanée calculée a pu être comparée aux mesures effectuées aux faibles vitesses de rotation (Fig. 6).

\subsection{Calcul des pertes}

Connaissant les vitesses de glissement instantanées et les forces de frottement [6], il est alors possible de calculer

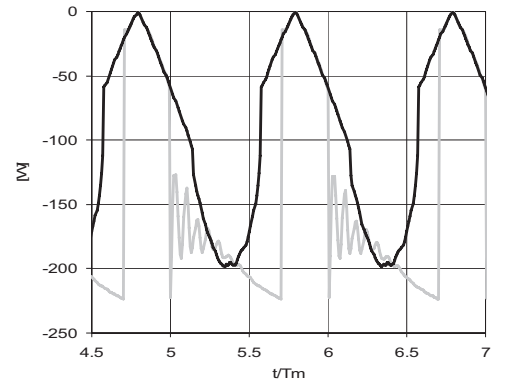

avec corrections sans correction

Fig. 7. Perte de puissance globale instantanée $(\mathrm{Cr}=1538 \mathrm{Nm}$; $\omega \mathrm{e}=600$ tr. $\mathrm{min}^{-1}$; denture droite).

Tableau 2. Caractéristiques des réducteurs modèles.

\begin{tabular}{|c|c|c|c|c|}
\hline $\mathrm{N}^{\circ}$ de réducteur & \multicolumn{2}{|c|}{1} & \multicolumn{2}{c|}{2} \\
\hline & pignon & roue & pignon & roue \\
\hline Nombre de dents & 42 & 155 & 20 & 20 \\
\hline Largeur de denture $(\mathrm{mm})$ & 205 & 200 & 30 & 30 \\
\hline Angle de pression $\left(^{\circ}\right)$ & \multicolumn{2}{|c|}{22,5} & \multicolumn{2}{c|}{20} \\
\hline Angle d'hélice $\left(^{\circ}\right)$ & \multicolumn{2}{|c|}{29} & \multicolumn{2}{c|}{0} \\
\hline Module $(\mathrm{mm})$ & \multicolumn{2}{|c|}{10} & \multicolumn{2}{c|}{10} \\
\hline Couple résistant $(\mathrm{Nm})$ & \multicolumn{2}{|c|}{150000} & 00 \\
\hline
\end{tabular}

directement la perte de puissance instantanée due au frottement. Il a été montré que les corrections de profils, qui correspondent à un enlèvement de matière sur les dents, peuvent réduire de façon significative les pertes. Par exemple, entre une denture non corrigée et une corrigée, une réduction de $50 \%$ de la perte moyenne a été calculée (Fig. 7). Ainsi, il apparaît comme très important de considérer ces corrections de dentures comme un moyen d'améliorer le rendement d'un réducteur.

\section{Calcul des pertes de puissance pour deux réducteurs modèles}

Les différents modèles sont appliqués pour calculer la part de chacune des pertes sur deux réducteurs modèles simple étage dont les caractéristiques sont données au tableau 2.

La figure 8 donne l'évolution des différentes pertes en pourcentage de la perte totale en fonction de la vitesse de rotation en entrée du réducteur.

Il apparaît tout d'abord que la proportion de chacune des pertes dépend grandement de la vitesse de rotation mais aussi de la géométrie des engrenages réducteurs. Ainsi, pour des engrenages de grands diamètres (réducteur $\mathrm{n}^{\circ} 1$ ), la perte par ventilation devient rapidement prépondérante du fait des vitesses périphériques beaucoup plus élevées. Par contre, pour des réducteurs de vitesses périphériques plus réduites (réducteur $\mathrm{n}^{\circ} 2$ ), les pertes par frottement à la denture restent prépondérantes même si la proportion diminue avec l'augmentation de la vitesse. 

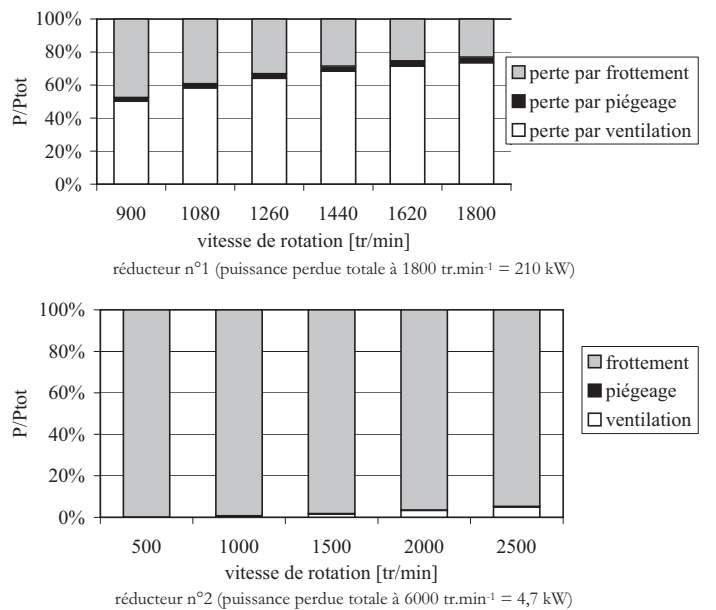

Fig. 8. Pertes de puissance en fonction de la vitesse de rotation sur le pignon.

Il est à noter que la faible proportion accordée aux pertes par piégeage du mélange air-lubrifiant dans l'espace inter-dents provient du fait que les calculs ont été menés pour le cas où seul de l'air serait piégé en pied de dent. Néanmoins, un premier constat nous montre que même si la proportion est relativement faible, une perte qui ne représente que $3,6 \%$ de la perte totale à 1800 tr.min ${ }^{-1}$ sur le pignon représente déjà $8 \mathrm{~kW}$ ce qui ne peut être négligé. De plus, ceci va induire des échauffements importants selon la largeur de la denture ce qui peut conduire à des dilatations différentielles importantes et donc provoquer des ruptures de denture qui à ces vitesses périphériques élevées représentent un risque très important.

\section{Conclusion}

Différents modèles pour évaluer les pertes de puissance dans les transmissions par engrenages dues à la ventilation de la denture, au piégeage du mélange airlubrifiant dans l'espace inter-dents et au frottement entre les dents ont été développés et validés sur des dispositifs expérimentaux. L'ensemble de ces modèles permet de prédire quel sera le rendement d'une transmission.

En appliquant ces modèles à deux réducteurs modèles, il ressort que la vitesse et la géométrie des dentures prises en compte influencent de façon importante les proportions de chacune des pertes. Ainsi, si la ventilation est un phénomène important pour des dentures de grandes dimensions, le frottement à la denture reste la perte dominante sur les réducteurs qui ont des vitesses de rotation faibles. Enfin, le phénomène de piégeage du mélange air-lubrifiant dans l'espace inter-dents qui représente une part faible sur l'ensemble des pertes, ne peut être négligé puisqu'il peut conduire à des dilatations différentielles engendrées selon la largeur de la denture et par conséquent à des surcharges sur les contacts.

\section{Références}

[1] Y. Diab, F. Ville, P. Velex, C. Changenet, Windage losses in high speed gears, Preliminary experimental and theoretical results, J. Mech. Design 126 (2004) 903-908

[2] P.H. Dawson, Windage loss in larger high-speed gears, Proc. ImechE 198A (1984) 51-59

[3] Y. Diab, F. Ville, H. Houjoh, P. Sainsot, P. Velex, Experimental and numerical investigations on the airpumping phenomenon in high speed spur and helical gears, Proc. I Mech. E Part C, J. Mech. Eng. Sci. 219 (2005) 785-800

[4] M.W. Rosen, The noises of two spur-gear transmissions, 1959, NOTS TP 2293, NAVORD Report 6569

[5] M.J. Pechersky, M.J. Wittbrodt, An analysis of fluid flow between meshing spur gear teeth, in Proc. 5th ASME Power Trans. Gearing Conference, 1989, Chicago, pp. 335-342

[6] Y. Diab, F. Ville, P. Velex, Prediction of power losses due to tooth friction in gears, Tribology transactions 49 (2006) 260-270

[7] G.H. Benedict, B.W. Kelley, Instantaneous coefficients of gear tooth friction, Tribology Transactions 4 (1961) 5970

[8] M. Vaishya, D.R. Houser, Modeling and measurement of sliding friction for gear analysis, Amer. Gear Manufacturers Association, 99 FTMS1 (1999) 1-20

[9] F. Ville, P. Velex, Introduction du frottement sur les dentures dans la simulation du comportement dynamique de transmissions par engrenages, XIV Colloque Vibrations, chocs et bruit, 16-18 juin 2004, Lyon, CD-Rom, 12 p.

[10] S. Baud, P. Velex, Static and dynamic tooth loading in spur and helical geared systems-experiments and model validation, ASME, J. Mech. Design 124 (2002) 334-346

[11] Y. Diab, Analyse des pertes de puissance dans les transmissions par engrenages à grande vitesse. Applications aux réducteurs industriels et aux machines textiles, Thèse, INSA Lyon, 2005, 144 pages, disponible sur : http://docinsa.insa-lyon.fr/these/pont.php?id=diab 